\title{
Kurzbericht zum Perspectives Workshop: Digital Social Networks
}

\author{
Andreas Henrich
}

Eingegangen: 16. Februar 2010 / Angenommen: 4. März 2010 / Online publiziert: 27. April 2010

(C) Springer-Verlag 2010

Vom 24. bis zum 29. Januar 2010 fand auf Schloss Dagstuhl ein Perspectives Workshop zum Thema ,Digital Social Networks“" statt (http://www.dagstuhl.de/10041). Die Initiative zu diesem Workshop ging von François Bry (LMU München), Clemens Cap (Universität Rostock), Ingo Dahm (Microsoft Deutschland GmbH), Julia Maintz (Maintz Research) und Sebastian Schaffert (Salzburg Research Forschungsgesellschaft) aus. Die insgesamt 28 Teilnehmerinnen und Teilnehmer des Workshops diskutierten in dieser Woche aktuelle Entwicklungen und zukünftige Perspektiven digitaler sozialer Netze aus der Perspektive der Informatik und aus einer interdisziplinären Perspektive, die für das betrachtete Gebiet unabdingbar ist. Drei wichtige Bereiche, die dabei diskutiert wurden, waren das Begriffsverständnis, die Herausforderungen und Möglichkeiten sowie die sich ergebenden Forschungsfelder. Die folgenden Ausführungen hierzu orientieren sich an den Diskussionen und den Ergebnispräsentationen der einzelnen Arbeitsgruppen.

\section{Das Begriffsverständnis}

Im Begriffsverständnis wurde der Terminus ,digitale soziale Medien“ hervorgehoben. Diese nutzen Informations- und Kommunikationstechnologien für Benutzer und (potentiell entstehende) Communities, um kollaborativ Inhalte zu erzeugen oder auszutauschen und - ganz allgemein - zu interagieren. Digitale soziale Medien erleichtern und stärken

\section{A. Henrich $(\bowtie)$}

Fakultät für Wirtschaftsinformatik und Angewandte Informatik, Lehrstuhl für Medieninformatik,

Otto-Friedrich-Universität Bamberg, 96052 Bamberg,

Deutschland

e-mail: andreas.henrich@uni-bamberg.de

url: http://www.uni-bamberg.de/minf/ die soziale Interaktion durch Überwindung physikalischer Grenzen in der Kommunikation (wie Entfernung und Synchronität) und Abmilderung menschlicher Einschränkungen (wie die Zahl der Menschen, mit denen man Beziehungen pflegen kann). Digitale Medien bieten damit Chancen für soziale Interaktionen, die ohne sie nicht möglich wären.

Die Beispiele für soziale Online-Medien und digitale soziale Netzwerke sind vielfältig. Sie umfassen Websites zur Pflege sozialer Netzwerke (wie Facebook, StudiVZ, LinkedIn und Xing), Blogs, Content-Sharing-Websites (wie Flickr, YouTube und last.fm), Bewertungen (wie epinions.com), soziale elektronische Spiele (wie Second Life), Social Bookmarking- (oder Tagging-)Plattformen (wie delicious), Backchannels (wie Twitter), Wikis und „open innovation" Plattformen (wie InnoCentive). Im Google-WaveProjekt wird die traditionelle E-Mail zu einem sozialen Medium umgestaltet. Obwohl digitale soziale Medien in ihrer heutigen Form ein recht junger Trend sind, haben sie eine sehr große Verbreitung - insbesondere, aber nicht nur, bei jüngeren Menschen - gefunden. Durch ihre Rückwirkung auf die Mediennutzung insgesamt (Zeitungen, Fernsehen, ...) und die Problematik der Offenlegung privater Daten sind digitale soziale Medien in der öffentlichen Diskussion. Auf der anderen Seite sind mit ihnen auch große Hoffnungen im Bereich des innovativen Wissensmanagements oder gar der Stärkung der Demokratie verbunden.

Unbestritten scheint, dass soziale Netze die Kommunikationsgewohnheiten verändern werden oder zumindest das Potential dazu haben. Sie passen sehr gut zu den Kommunikationsbedürfnissen im Zeichen erhöhter Mobilität und zunehmender Globalisierung. Durch die individuelle Möglichkeit Informationen öffentlich zugänglich zu machen, entstehen aber auch Rechtsfragen und Fragen der Privatsphäre, die mit dem jeweiligen Landesrecht kaum zu regeln sind. 


\section{Herausforderungen und Möglichkeiten}

Die Herausforderungen und Möglichkeiten digitaler sozialer Medien liegen in sozio-kulturellen, ökonomischen, politischen und technischen Bereichen. Würden die in einem dieser Bereiche tätigen Forscherinnen und Forscher glauben, die Fragestellungen allein verfolgen zu können, würde dies zwangsweise zu einseitigen Ansätzen führen.

Auf der sozio-kulturellen Ebene bieten digitale soziale Medien neue Möglichkeiten die eigene Identität darzustellen oder auch zu verändern. Auch die Möglichkeiten der Partizipation werden in qualitativer und quantitativer Hinsicht ausgeweitet. Auf der anderen Seite steht die Gefahr, dass Personen, die - aus welchen Gründen auch immer - keine Möglichkeiten des Zugangs zu diesen Medien haben, ausgegrenzt werden. Das Stichwort des „digital divide" steht für dieses Problemfeld. Hinzu kommt, dass durch mobile Endgeräte und Pervasive Computing digitale soziale Medien potentiell allgegenwärtig sind. Aus ökonomischer Sicht sind Geschäftsmodelle für die Betreiber digitaler sozialer Netze relevant. Hier ist auch der Zusammenhang zwischen potentiellen Geschäftsmodellen und den intendierten Kommunikationsformen zu beachten. Ferner gewinnen digitale soziale Medien in der Unternehmenskommunikation und im Wissensmanagement von Unternehmen eine immer größere Bedeutung. Daneben entstehen durch die Kollaboration in digitalen sozialen Medien Wirtschaftsgüter von hohem Wert. Man denke z. B. an Wikipedia oder Foto-Sharing-Plattformen. Schließlich hat die zunehmende Bedeutung digitaler sozialer Medien Rückwirkungen auf etablierte Geschäftsmodelle, die sie ergänzt oder erschwert.

Im politischen Bereich stellen sich neben Fragen der Privatsphäre, des Datenschutzes und z. B. des Urheberrechts auch Fragen der freien Meinungsäußerung oder der Zensur. Nicht zuletzt haben die letzten Wahlen verdeutlicht, dass digitale soziale Medien in ihrer Bedeutung und Geschwindigkeit große Auswirkungen auf die öffentliche Meinung haben und so auch im Wahlkampf und in der politischen Diskussion einen Wandel herbeiführen.

Im technischen Bereich ist die Entwicklung digitaler sozialer Medien natürlich mit anderen Entwicklungen wie dem „Internet der Dinge“ oder dem „Ubiquitous Computing“ verknüpft. In den Vordergrund der Diskussion treten aber immer wieder Fragen der Interoperabilität zwischen den verschiedenen Angeboten. Die Vielfalt der Angebote ist sicher für eine Zeit auch die treibende Kraft der Innovation gewesen, sie könnte aber auf Dauer zu einem großen Hemmnis werden, wenn es nicht gelingt, die Interoperabilität zu steigern. Weitere Herausforderungen sind sicher im Bereich der Skalierbarkeit und der Personalisierung zu sehen.

\section{Forschungsfelder}

Digitale soziale Medien integrieren als sozio-technische Systeme sozio-kulturelle und technische Innovationen. Daher sind die verbundenen Forschungsfelder inhärent interdisziplinär. Entsprechend war auch der Workshop interdisziplinär besetzt - allerdings mit einem deutlichen Übergewicht in der Informatik. Die Frage nach wichtigen Forschungsfeldern wurde daher mit der Frage hinterlegt, welche Technologien und Methoden die Informatik zur weiteren Entwicklung digitaler sozialer Medien beitragen kann. Insgesamt wurden auf einer hohen Abstraktionsebene vier Forschungsfelder identifiziert: „Society and Economy“, „Architecture and Infrastructure“, „Services and Applications“ sowie „Trust, Privacy and Security“.

Im Bereich von Gesellschaft und Wirtschaft werden Schwerpunkte im Bereich der Bildung im Umgang mit Medien, im Bereich der (supranationalen) rechtlichen Rahmenbedingungen, der Geschäftsmodelle oder der Analyse des Sozialverhaltens gesehen. Für die Architekturen sind Stichworte wie Skalierbarkeit und insbesondere Interoperabilität von großer Bedeutung. Dabei können neben zentralen und verteilten Architekturen auch Peer-to-Peer-Ansätze an Gewicht gewinnen.

Dienste für digitale soziale Medien werden im Monitoring und in der Analyse ebenso gesehen wie in einem allgemeineren ggf. mit Governance zu beschreibenden Feld. Dabei geht es darum, die technischen und sozialen Regeln eines digitalen sozialen Netzwerks zielgerichtet gestalten zu können. Einen weiteren interessanten Bereich bilden ,sozial informierte“ Algorithmen, die das gemeinschaftliche Wissen in einem sozialen Netz nutzen, um z. B. Suchergebnisse zu verbessern oder Vorschläge zu generieren. Sozial informierte Algorithmen sind aber auch in der Routenplanung und anderen Aufgabenstellungen von Bedeutung. Bei all dem wird für die breite Akzeptanz digitaler sozialer Netzwerke die zufriedenstellende Beantwortung der immer lauter werdenden Fragen im Hinblick auf Vertrauenswürdigkeit, Privatsphäre und Sicherheit eine entscheidende Rolle spielen. Auch dies ist wieder ein Bereich, in dem technologische und soziale Aspekte zusammenwirken müssen.

\section{Wie geht es weiter?}

Derzeit schreiben die Teilnehmerinnen und Teilnehmer des Workshops an einem „Manifest“ zum Thema, das in den nächsten Wochen fertiggestellt werden soll. Dieses „Manifest" wird über die Webseite zum Workshop (http://www. dagstuhl.de/10041) zugänglich gemacht werden. Auf dieser Webseite finden sich auch die Kontaktdaten der Organisatoren des Perspectives Workshops.

Ich persönlich habe den Workshop in Dagstuhl sehr genossen. Die vielen verschiedenen Blickwinkel auf digitale 
soziale Netze haben mir geholfen, meine persönliche Wahrnehmung des Themas aus dem Blickwinkel des ,Information Retrieval“ in einen größeren Kontext zu stellen. Dadurch haben sich interessante neue Fragestellungen und Kontak- te ergeben. Ich möchte diese Gelegenheit daher nutzen, den Organisatorinnen und Organisatoren zu danken, und ich denke, dass eine Folgeveranstaltung in ca. zwei Jahren sicher eine gute Idee wäre. 